of the plant and treatment. Samples submitted to the laboratory are often poorly identified with approximately $50 \%$ of the database entries having little description beyond the category. This is particularly the case for the pasture species. Information is usually lacking on stage of growth and how the sample might represent either the pasture on offer or that selected by the animal and hence inclusion of these pasture samples might be considered inappropriate.

Depending on the feed type and the submitter's request, the analyses performed on a feedstuff could include dry matter, ash, total nitrogen, gross energy, amino acids, crude fat, fatty acids, crude fibre, neutral detergent fibre, acid detergent fibre, lignin, in vitro digestibility (measured using rumen fluid), water soluble carbohydrate, starch, amylose, beta glucans, $\mathrm{Ca}, \mathrm{P}, \mathrm{Na}$ and $\mathrm{K}$.
Conclusions: The Queensland Animal Science database contains nutritional data for a large number of tropical and sub-tropical animal feeds that potentially could be included in an international, public domain, feeds database. Because detailed information is lacking for the majority of 'pasture' samples, contributions might best be limited to concentrates, hay and silage. To ensure best quality of the data and the scientific outcomes, resources are needed to scrutinise the information in the database e.g. to confirm descriptions and to confirm the matching of formats and nomenclature.

\section{References}

Göhl B 1975. Tropical Feeds, Feeds Information Summaries and Nutritive Values (Unedited Version). FAO, Rome.

\title{
Tables of nutritive value for farm animals in tropical and Mediterranean regions: an important asset for improving the use of local feed resources
}

\author{
Gilles Tran ${ }^{1}$, Valérie Heuzé ${ }^{1}$, Denis Bastianelli ${ }^{2}$, Harry Archimède ${ }^{3}$ and Daniel Sauvant ${ }^{1,4}$ \\ ${ }^{1}$ Association Française de Zootechnie, 75231 Paris Cedex 05, France; ${ }^{2}$ CIRAD, Systèmes d'élevage et produits animaux, 34398 Montpellier Cedex 05, France; \\ ${ }^{3}$ INRA, UR143 Unité de Recherches Zootechniques, 97170 Petit-Bourg, France; ${ }^{4}$ AgroParisTech, UMR 791 MoSAR, 75231 Paris Cedex 05, France \\ Email: gilles.tran@gmail.com
}

\section{Introduction}

The optimisation of feed resources is of paramount importance and requires accurate feed values. Tables of feed composition and nutritive value are used to formulate diets that meet animal requirements at the lowest cost, thereby improving production results and farm productivity. In emerging and developing countries, and particularly those in tropical and Mediterranean areas, the demand for animal products has been steadily growing in the past decades, and so has the need for accurate feed information. However, even though many research data are published every year, they are rarely summarized in an easily available form, and users in these regions must often rely on data obtained in temperate countries or use obsolete or incomplete sources.

The Tables of nutritive values for farm animals in tropical and Mediterranean regions is a current project of INRA, CIRAD and AFZ (with partial support from the FAO) that aims to produce comprehensive tables for feed users in these areas. The tables are planned for release in 2013, either in paper or electronic form. In addition to AFZ staff, the project involves INRA and CIRAD researchers, and research organizations from other countries.

\section{Objectives}

The main objective is to create a compendium of up-to-date information on feed materials available to users in tropical, subtropical and Mediterranean regions. The project focuses on the following information:

- Feed nomenclature and descriptions: names, physical aspect (including pictures), availability, processes and environmental impact.

- Recommendations by livestock species, including cautionary information.

- Composition and nutritive values derived from feed databases and scientific literature.

For local users, the following benefits should be expected:

- Better identification, qualification and quantification of local feed resources.

- Better opportunities for livestock in sustainable integrated farming systems.

- Better use of local feeding practices and less reliance on imported techniques and feed materials.

- In the near future, it will also be possible to use the tables' framework for quantitative environmental data. 
For researchers, the tables should:

- Help to promote collaboration between teams working on tropical and Mediterranean animal feeding.

- Help to identify areas of incomplete knowledge, thereby stimulating needed research.

\section{Methodology}

For each feed material or family of feed materials, we will use the following methodology:

- Research and identification of relevant databases and scientific literature

- Collection and summarizing quantitative and qualitative feed data

- Establishing representative and consistent vectors of chemical and nutritive values. For large families of feedstuffs, the methods of meta-analysis will be used.

- The project is managed through a collaborative website that is editable on-line by the contributors. A database contains the composition and nutritive data, equations and calculation processes.

\section{Current results}

- A database containing more than 2 million raw data has been established.

- Specifications have been drawn up for researching information and writing datasheets.

- Collaborations have begun with Gembloux Agrobiotech (Belgium), the Hassan II Institute (Morocco) and the University of Cordoba (Spain). Other institutions are interested in participating.

- A first batch of 100 datasheets will be produced by the end of 2010 .

\section{To be done}

- Establishing a formal methodology for calculating final table values

- Generation of approximately 200 datasheets per year for the next 3 years.

- Finding new partners in other countries in order to increase the breadth of both data collection and expertise

- Development of a website in partnership with the FAO. The website content will be maintained on a permanent basis to provide updated information to feed users.

\title{
Sub-saharan African feed database
}

\author{
Alan J. Duncan ${ }^{1}$, Bruno Gerard ${ }^{2}$ and Katrien Descheemaeker ${ }^{1,3}$ \\ ${ }^{1}$ International Livestock Research Institute, PO Box 5689, Addis Ababa, Ethiopia; ${ }^{2}$ CGIAR Systemwide Livestock Programme, ILRI, PO Box 5689, Addis Ababa, \\ Ethiopia; ${ }^{3}$ Current address : CSIRO, Ecosystem Sciences, BMP 2, Glen Osmond, SA 5064, Australia \\ Email : A.Duncan@cgiar.org
}

SSA Feeds is a web-based database of nutritional parameters for a range of feeds commonly fed to livestock in Sub-Saharan Africa. The database is reasonably comprehensive and provides advanced search functions as well as allowing descriptive statistics for sub-sets of data to be generated. The database is a useful resource for extension, development and research agents to design scientifically-based rations for meat, dairy and draught animals of small-scale African farmers. The database also contains useful nutritional data for development of nutritional components of farm household models such as lifesim (http://vs/p.org/models/).

\section{What is SSA Feeds?}

SSA Feeds is a database of nutritive values of feeds commonly fed to livestock in Sub-Saharan Africa. The database is freely available on the internet (http://vslp.org/ssafeed/) and is widely used. The database contains information on 459 livestock feed types from 14 countries in sub-Saharan Africa. The data is based on 14571 samples analysed over the years in the nutrition labs of the International Livestock Research Institute. An additional database provides nutritional data for Ethiopian feeds derived from the literature including references to source material.The database has strong search capabilities allowing searches by country, feed type, genus and species and also a user-friendly free search facility. The web-based interface also allows information from a range of feed samples to be summarised using descriptive statistics including mean values, standard deviation, minimum value and maximum value. Raw data can be exported to spreadsheets for further data analysis.

\section{What parameters?}

The database includes a comprehensive range of measures of nutritive value including the «Van Soest fibre» parameters, NDF and ADF as well as in vitro dry matter digestibility (IVDMD). Metabolizable energy values are estimated from IVDMD and net energy values for maintenance, gain and lactation are also calculated within the database. Crude protein values estimated as $6.25 \times$ Kjeldahl nitrogen concentrations are also presented. Finally a range of important trace element concentrations are available.

\section{Who is it useful for?}

The data presented in the database is designed to enable extension, development and research agents to design scientifically-based and best-cost rations for meat, dairy and draught animals of small-scale African farmers. The data will also be useful for research purposes, in particular for parameterizing nutritional components of farm household models such as lifesim (http://vslp.org/models/). The database fills a gap in global feed data since most other data sources neglect the feeds which predominate under Sub-Saharan African conditions. 